\title{
Preparing for medical education after the COVID-19 pandemic: insightology in medicine
}

\author{
Yon Ho Choe ${ }^{1,2}$
}

${ }^{1}$ Department of Pediatrics, Samsung Medical Center, and ${ }^{2}$ Department of Medical Humanities, Sungkyunkwan University School of Medicine, Seoul, Korea

It is necessary to reflect on the question, "How to prepare for medical education after coronavirus disease 2019 (COVID-19)?" Although we are preparing for the era of Education 4.0 in line with the 4th industrial revolution of artificial intelligence and big data, most measures are focused on the methodologies of transferring knowledge; essential innovation is not being addressed. What is fundamentally needed in medicine is insightful intelligence that can see the invisible. We should not create doctors who only prescribe antispasmodics for abdominal pain, or antiemetic drugs for vomiting. Good clinical reasoning is not based on knowledge alone. Insightology in medicine is based on experience through Bayesian reasoning and imagination through the theory of mind. This refers to diagnosis of the whole, greater than the sum of its parts, by looking at the invisible using the Gestalt strategy. Identifying the missing process that links symptoms is essential. This missing process can be described in one word: context. An accurate diagnosis is possible only by understanding context, which can be done by standing in someone else's shoes. From the viewpoint of medicine, Education 4.0 is worrisome because people are still clinging to methodology. The subject we should focus on is "human", not "artificial" intelligence. We should first advance the "insightology in medicine" as a new paradigm, which is the "essence" that will never change even when rare "phenomena" such as the COVID-19 outbreak occur. For this reason, we should focus on teaching insightology in medicine, rather than teaching medical knowledge.

Key Words: Insightology in medicine, COVID-19, Medical education, Insight quotient

\section{Introduction}

Anyone interested in medical education during the coronavirus disease 2019 (COVID-19) crisis must have had the following concern: "How do we prepare for medical education after COVID-19?" It is the most basic question, and an important topic for medical educators.

At the end of the 17th century, Europeans first entered the Australian continent and discovered a black swan. The discovery was a moment that presented both surprise and emptiness to explorers. They had thus far believed swans to be only white, which in a single instant became fiction. Unpredictable and extreme situations can happen at any time. Taleb [1] presented a new perspective on the world in his book "Black swan." Rare things can happen, and we should be prepared for them. The human brain has a tendency to immerse in
Received: August 6, 2021 - Revised: August 6, 2021 - Accepted: August 10, 2021 Corresponding Author: Yon Ho Choe (https://orcid.org/0000-0003-1525-7688)

Department of Pediatrics, Samsung Medical Center, Sungkyunkwan University School of Medicine,

81 Irwon-ro, Gangnam-gu, Seoul 06351, Korea

Tel: +82.2.3410.3527 Fax: +82.2.3410.0043 email: i101016@skku.edu
Korean J Med Educ 2021 Sep; 33(3): 163-170

https://doi.org/10.3946/kjme.2021.196

eISSN: $2005-7288$

(C) The Korean Society of Medical Education. All rights reserved. This is an open-access article distributed under the terms of the Creative Commons Attribution Non-Commercial License (http:// creativecommons.org/licenses/by-nc/3.0/), which permits unrestricted non-commercial use, distribution, and reproduction in any medium, provided the original work is properly cited. 
novelty when it sees a phenomenon for the first time. This is because of the limitations of the three thoughts that humans are born with. First is the thinking of sight. In his 1620 book, "New organon", Francis Bacon said, "Human thinking stops at sight. Therefore, humans cannot afford to discern what cannot be seen with their own eyes [2]." We only believe in what we see, and are very vulnerable to seeing the invisible. The area of the brain responsible for imagination is identical to that which controls senses such as sight and hearing; thus, if one sees and imagines something, finding anything other than what we have experienced for ourselves is difficult. The second is a matter of knowledge thinking. If there is a big difference in the level of knowledge when two persons are talking, one will feel very frustrated. Differences in knowledge are important, but even more problematic is that we do not know the depth of our own knowledge. While we think we know about things and people around us, we do not know much in-depth. We only think we know them well because we are used to them. The third is the thinking of satisfaction. Herbert Simon was awarded the Nobel Prize in Economics for discovering the limitations of cognitive ability called "bounded rationality". When the best solution is not presented for decision-making, humans stop halfway through "satisficing". By using only limited information, that is, our own experience and knowledge, we stop thinking better, and draw our own satisfactory conclusions [3].

Due to these three limitations, humans tend to be content with seeing and interpreting new phenomena within the boundaries of their knowledge, rather than taking deep and steady actions to find their essence. The COVID-19 outbreak was a black swan. Black swans such as pandemic infectious diseases and natural disasters are rare, but will continue to occur in the future. We must reflect whether we will be engulfed in the situation and panic whenever the black swan appears. Going back to the question, "How to prepare for medical education after COVID-19," we must ask ourselves if we are ready to answer. How can we discuss how to respond every time the situation changes, without giving an essential answer? So, I would like to remove the "after COVID-19" part of the question. Once again, we have to seriously consider how to prepare for medical education.

\section{Why patients should not be examined with medical knowledge}

$\mathrm{Na}$-young was born slightly small, weighing $2.9 \mathrm{~kg}$ at birth. Her parents began to worry about the baby being underweight when the postpartum care center stated that the baby did not seem to eat as much as others. The parents were deceived by the false information that feeding issues may occur if the baby suffers from tongue-tie. At 6 months, Na-young underwent tonguetie surgery, but she was still not eating a sufficient amount. Disliking baby food, $\mathrm{Na}-$ young had nausea and vomiting when she was 9 months old. Frightened, her parents took $\mathrm{Na}-$ young to a gastroenterologist. A university hospital doctor who heard the story, "The child is not eating well and is not growing. These days, she often has nausea and vomits," began the examination. After gastroscopy, gastroesophageal reflux disease and allergic gastroenteritis were suspected. The mother was relieved, thinking "Ah, it was because she was ill." Accordingly, she gave her daughter the prescribed medication for 12 weeks, but Na-young did not get better. On a restricted diet because of food allergies, the child stopped eating and vomited further. Finally, Na-young was brought to me. After analyzing the situation, it was determined that $\mathrm{Na-young}$ was not ill. Due to the parents' serious concerns, unnecessary tests 
were conducted and medications given. In fact, $\mathrm{Na}$-young was a picky eater [4].

Children who have a small appetite and are fussy are often observed. Since these children are not very interested in eating, parents fear they will not grow up healthy. There is a high probability that one of the parents was also picky when young. For a sensitive mother, the fact that the child is not growing is her own loss. To avoid this fear of loss, the mother takes certain actions, such as going to the hospital, changing formula several times, and even performing tongue-tie surgery. However, there is one more issue that should not be overlooked. When the mother visited the university hospital, the doctor recommended an endoscopy, and diagnosed $\mathrm{Na}-$ young with reflux esophagitis and allergic gastroenteritis. The doctor diagnosed this by linking it to the child's symptoms, and gave the child an unnecessary drug for 12 weeks. The same action bias of doctors who reacted only to superficial phenomena eventually resulted in a kind of iatrogenesis. My prescription for Na-young was simple; I recommended not to give the child any medicine, and rather give her the right to decide on eating. It meant giving her freedom to eat. Picky children who do not eat well become able to eat everything as they go through puberty and enter university. If parents force children to eat and put stress on them for not eating, the children develop oral aversion. Oral aversion is mainly caused by the guardian's obsession and loss aversion. One's future is someone else's present. Everyone will change, but people make the error of "presentism", which determines the future based on what is happening right in front of them [5]. Na-young was observed for 6 months, and gained weight well. Doctors must first be able to read the minds of patients and their families. In addition, we must not forget that we will miss the proper diagnosis and treatment if we examine patients with only medical knowledge.

\section{What is innovation in medical edu- cation?}

We are aware that there are problems with current medical education. Although we are preparing for the Education 4.0 era in line with the 4th industrial revolution of artificial intelligence and big data, most measures are methodologically biased, and do not seem able to address essential innovation. In fact, the COVID-19 crisis has only brought about a change in the educational methodology, not the fundamental mission of education. The medical education paradigm has not changed, perhaps because we have not yet fully sensed the crisis.

If we had to choose one of the most cited books of the 20th century except the Bible, which one would it be? One would surely be Thomas Kuhn's "The structure of scientific revolutions", which Ian Hack calls a "great book". First published in 1962, it contains excellent insights into the development of science as well as the word "paradigm". Thomas Kuhn was the first to introduce the everyday term "paradigm" of "paradigm shift", in his book, which we use commonly today. Kuhn called the science studied at that time "normal science", and stated that normal science is characterized by a paradigm. That is, normal science does not aim at novelty, it aims at clarifying the status quo. Under a paradigm, people think, experiment, and reflect. But one day, the things people have known for a long time do not fit, and start to seem strange. People think, "Why is there an anomaly?" This is the law that leads to the emergence of scientific discovery. As an unexpected anomaly repeats, "crisis" is reached. When the anomaly reaches a point it can no longer be healed, it does not end with denying the 
existing theories, but new theories and new studies replacing them appear. This is a "paradigm shift". "When a specific phenomenon is an anomalous phenomenon that is difficult to explain, while it becomes a natural phenomenon such as a law on one side, how can these two be compared on a reasonable basis?" Kuhn called this "incommensurability". To Newton, the theory of special relativity would not make sense, and to Einstein, classical mechanics would seem insufficient, but both theories are new paradigms that have changed the paradigm of the previous era [6].

The normal science we are examining under the current paradigm of medical education is Education 4.0 represented by evidence-based medicine and artificial intelligence. This article will discuss a new paradigm of medical education: insightology in medicine based on humanism. While medical education of humanism has always been practiced, real training for insight has not been carried out. In addition, there is an incommensurability between the two.

\section{Insightology in medicine}

The biggest change brought about by the COVID-19 crisis is the appearance of non-face-to-face online lectures. However, I do not think it matters much whether the method of education delivery is faceto-face or non-face-to-face. In medical education, the two methods should be complementary, and either way is appropriate as long as the message gets delivered. The greater difficulty is that our bias toward the method of delivering knowledge may not allow us to identify the essence of the incident. Now, knowledge is at a stage where artificial intelligence can fully replace it. A human doctor's job is to make the right diagnosis, and take care of patients and their families. While diagnosis may also be performed by artificial intelligence, machine learning without understanding the context cannot match with the medical science of humanism. The true essence of medical education is thus humanism.

To date, human intelligence has been described as intelligence quotient (IQ) and emotional quotient (EQ). Furthermore, Gardner [7] introduced multiple intelligences such as music, movement, logical mathematics, language, space, human-friendliness, self-reflection, and nature-friendly. However, we often see people around us who are not necessarily smarter, but are on the road to success. What abilities make them successful? In the bigger picture, their success can be expressed in one word: "Insight". All successful people in modern society have this insight in common. Like EQ, insightful intelligence will also be difficult to measure, but I will call it insight quotient (InQ). The dictionary definition of insight is "seeing through things with keen observation". My interpretation of this definition is that keen observation is the domain of IQ, and seeing through things is the domain of EQ. In other words, InQ is at least the sum of IQ and EQ. As an equation, this would be expressed as IQ+EQ<InQ. Based on IQ and $\mathrm{EQ}$, InQ means integrated intelligence that sees, feels, and acts in the world. IQ and EQ are already included by default in InQ. Insightful intelligence is the ability to read the context of events around oneself, and see the invisible [6].

"We must not perceive the patient based solely on medical knowledge," I always tell my students. Rather than medical knowledge, we should teach how to learn. For example, I do not educate students to become doctors who would simply prescribe antiemetics for patients suffering from vomiting. The etiology might not be gastrointestinal alone, but it may be cerebral or psychological, and we can identify unexpected diseases by taking into account symptoms other than vomiting. I 
teach students to communicate with patients and their family, and to astutely inquire about any environmental changes experienced. Therefore, I encourage students to consider a broad variety of studies: psychology, to understand others' thoughts; engineering, to form the foundations of medical technology; and economics, to carve something out for themselves. Furthermore, we need to prepare for the future. As Yuval Harari, author of "Homo Deus" writes, "Everything changes." This change projects a near future with artificial intelligence, which Ray Kurzweil depicts in "The singularity is near". we must develop the ability to penetrate artificial intelligence, even though machine learning will eventually replace the role of human doctors. Society demands an education that enriches doctors, and ensures they can continue to shine even if dataism does take over, as Harari states. Such an education is possible if based on humanism. Artificial intelligence must develop together with humanism, and teachers and learners must embrace it with insight. I believe that raising InQ beyond IQ and EQ should be the frame of medical education. Only then will doctors from the people be born for the people [6].

Kurtz [8], who has consistently studied contextual intelligence, defined it thus: "Selecting and executing the best action by recognizing the various variables raised in a given situation and accurately discriminating the

Fig. 1. Three-Dimensional Thinking Model of Contextual Intelligence

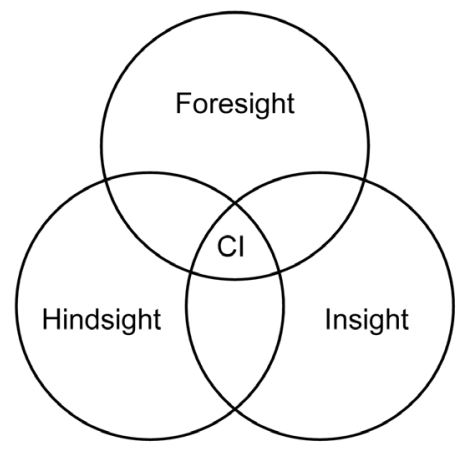

Cl: Contextual intelligence. differences between different courses of action." In addition, Kurtz [9] diagrammed contextual intelligence as a three-dimensional thinking model, shown in Fig. 1. This is very practical and helps to put it into action. Hindsight is the ability to use one's experience, and foresight is the ability to predict possible plans for the future. According to Kurtz [9], insight is the ability to know what is influencing the present moment. Moreover, he notes the interrelationship of these three, and explains that insight equals hindsight plus foresight. In my opinion, contextual intelligence is identical to insightful intelligence [6].

To help understand this schematic figure concretely, I will change the terms used. As shown in Fig. 2, hindsight becomes experience, foresight becomes imagination, and insight becomes seeing the absence [6]. In other words, absence can be seen using one's own experience and imagination. Experience is the past, and we make judgments about the present and the future based on our experiences. Humans weigh probabilities when they have to decide their actions ahead of a situation. There are two ways to calculate probability. One is Fisherian statistics, which measures the frequency, and the other is Bayesian statistics, which estimates the posterior probability by making additional observations based on prior probability. When a baby experiences pain and has

Fig. 2. Insightful Intelligence That Reads the Invisible Through Experience and Imagination

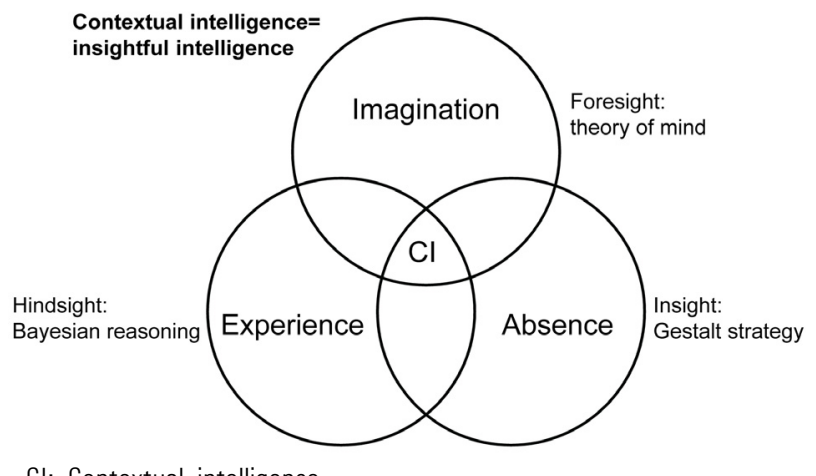

Cl: Contextual intelligence. 
a bad memory, the baby will react in a new way to avoid this experience in the future. Imagination is the future. This is also a way of thinking specialized in humans. When we talk to others, we read their minds. This is possible because only humans have a "theory of mind"; our brains have mirror neurons that enable imitation. By observing what the other person says and does, we create our own imagination, which could be right or wrong. The more our thinking turns out to be right, the more our insight grows. Insight refers to the perception of the world through Gestalt strategy. Finding out the "missing process" that connects the parts, rather than just focusing on the parts, is essential. To describe the missing process in one word, it is context. Understanding this context allows us to read the whole, which is greater than the sum of its parts. The easiest way to understand context is to see from the "other's point of view". By putting oneself in someone else's shoes, listening to that person's experience, and imagining based on that person's experience, one suddenly sees things one could not see earlier. Contextual intelligence or insightful intelligence plays a role in the process of integrating all of this to see the whole, including anyone's experience or imagination [6].

On January 8, 2011, at around 10:00 AM, a member of the United States' House of Representatives, Gabrielle Giffords, the next generation prospect of the Democratic Party in Tucson, Arizona, USA, collapsed after being shot down while speaking to voters. She was taken to the emergency center of the University of Arizona Hospital suffering a head-piercing injury. Emergency surgery was decided upon, and the operating surgeon was Peter Rhee, head of the emergency center. Born in Seoul in 1961, he became an army surgeon in the United States. He was a veteran of gunshot wounds, and came across numerous traumatic surgeries while serving in the military for 24 years. Prior to surgery, Peter Rhee responded to a press request for an interview; everyone wondered whether Giffords would live or die [6].

"Is Giffords dead?" "No, she's still alive." "There are reports on CNN that she has already died." "I am going into surgery now. It is true that the damage is significant, but there is a possibility of survival." The reporters spoke noisily, "Are there any chances of survival? What percentage?" Peter answered this question briefly and firmly, "101 percent." "Yeah? 101 percent? What's wrong with your mind?" The reporters were bewildered since it was a head-piercing wound. A few days later, the miraculous news came that Giffords was awake. At the doctor's instructions, she responded by raising two fingers of her left hand. Headlines immediately praised Peter Rhee, and he was again interviewed.

"You predicted survival at 101 percent, how did you do that?" Peter replied calmly, "When I first went to the emergency room, it was strange. I watched her, while unconscious, gently grab the fingertip of the fellow doctor. It was a very unusual reaction I had never experienced with a patient with a gunshot wound to the head. At that moment, I was sure that Giffords would live [10]."

A gunshot wound to the head is in fact a death sentence. However, Rhee showed 101 percent confidence. Let us read Peter's mind in that situation. "It is the first time a person with a gunshot wound to the head holds the doctor's hand.. I've never had a gunshot wound to the head like this before." Peter quickly reviewed his clinical experience, and organized his hindsight. "I'd carry out the surgery sooner rather than later." Peter must have imagined where and how to approach the surgery to minimize brain damage. Determining the optimal treatment for this is foresight. "Surely Giffords will come out alive." This was the moment his insight turned into a confident prediction. Through the experience of the past, which is hindsight, 
and the imagination for the present and the future, which is foresight, Peter Rhee put his insightful intelligence to good use [6]. This is the use of insightology in medicine.

\section{Conclusion}

In "The Fourth Education Revolution" of Seldon et al. [11], the items of Education 4.0 are as follows: artificial intelligence; neuroscience and cognitive science; voice and image recognition; robotics; virtual reality, augmented reality, mixed reality; internet of things; big data and data storage; blockchain; collaborative learning; transhumanism.

These are very important areas, and are the details we need to most focus on in the future. However, what concerns the Education 4.0 of Seldon et al. [11] from the perspective of medical education is that people still cling to methodology. The subject we should be focused on is "human", not "artificial" intelligence. What makes humans superior to artificial intelligence is that they can read the context, are process-oriented, and can tell stories. In medical education, this insight should be taught. Professor Lee Byung Doo of Inje University Medical School noted that it takes 10 years after graduation for doctors to gain insight. While it is understood that insight naturally accumulates with time, do we have to wait for 10 years in this incredibly fast-changing era? Why don't we teach the methods of insight in medical education from the beginning?

Medical education in Korea is still in its infancy. Every year, we study and follow the topics raised by the World Federation for Medical Education, even as we know that we can never lead by imitating others. With its strength in clinical medicine, Korea should consider a lead in "insightology in medicine", which is the "essence" that will not change even if a rare "phenomena" such as COVID-19 occurs again. Korea has enough ability and qualification to begin learning and teaching insightology in medicine.

ORCID:

Yon Ho Choe: https://orcid.org/0000-0003-1525-7688

Acknowledgements: None.

Funding: Article processing charges were supported by the Korean Society of Medical Education.

Conflicts of interest: No potential conflict of interest relevant to this article was reported.

Author contributions: All work was done by Yon Ho Choe.

\section{References}

1. Taleb NN. The black swan. Cha IJ, translator. Paju, Korea: Dongnyuk Science; 2008.

2. Sandel A. Arguments against prejudice. In: Sandel A, ed. The Place of Prejudice. Lee J, translator. Seoul, Korea: Wisebury; 2015:47-110.

3. Wikipedia. Herbert Simon. https://ko.wikipedia.org/wiki/ \%ED\%97\%88\%EB\%B2\%84\%ED\%8A\%B8_\%EC\%82\%A C\%EC\%9D\%B4\%EB\%A8\%BC. Accessed July 29, 2021.

4. Choe YH. Intervention. In: Choe YH, ed. Embracing the Bad Memory. Paju, Korea: Book Pot; 2020:110.

5. Gilbert D. Predicting the future with the experience of the present. In: Gilbert D, ed. Stumbling on Happiness. Seo EG, Choi IC, Kim MJ, translators. Paju, Korea: Kimyeongsa; 2006:165-185.

6. Choe YH. Insightful intelligence. Paju, Korea: Book Pot. Forthcoming 2021.

7. Gardner $\mathrm{H}$. The core of theory of multiple intelligences. In: Gardner H, ed. Multiple Intelligences. Moon YR, Yoo 
KJ, translators. Paju, Korea: Woongiin Knowledge House; 2007:21-46.

8. Kurtz M. What is contextual intelligence? In: Kurtz M, ed. Contextual Intelligence. Park SC, translators. Seoul, Korea: Hyeonamsa; 2018:21-40.

9. Kurtz M. 3D thinking: a different orientation to time. In: Kurtz M, ed. Contextual Intelligence. Park SC, translators. Seoul, Korea: Hyeonamsa; 2018:167-184.

10. Hwang JB. Prologue. In: Hwang JB, ed. Talking Medicine with the Heart. Paju, Korea: Lee Dam; 2012:5.

11. Seldon A, Metcalf, T, Abidoye O. The Fourth Education Revolution. In: Seldon A, Metcalf, T, Abidoye O, eds. The Fourth Education Revolution: Will Artificial Intelligence Liberate or Infantilise Humanity. Yeom DK, Park JH, Hong CW, Kwon JH, Kim SH, Lim KS, translators. Seoul, Korea: Sungkyunkwan University Press; 2020:38. 